\title{
EL DISCURSO MÉDICO A PROPÓSITO DE LAS FIEBRES Y DE LA QUINA EN EL TRATADO DE LAS CALENTURAS (1751) DE ANDRÉS PIQUER
}

\author{
Marcelo Frías Núñez*
}

Dpto. Historia de la Ciencia, IH, CSIC, Madrid

\section{RESUMEN}

En el presente trabajo analizamos la figura de Andrés Piquer, aportando una propuesta de análisis personal sobre su discurso médico, a partir de la edición de 1751 del Tratado de las Calenturas, utilizando como ejes reflexivos las alusiones a las fiebres y a la quina, y apoyándonos también en referentes españoles y europeos — con especial presencia del medio francés-. Así, nos encontramos con un Piquer que apuesta claramente por la observación y la experiencia como base del conocimiento médico. Consideramos esta propuesta como un acercamiento necesario que permita abordar, en un momento posterior, las siguientes ediciones, con el horizonte final de un análisis comparativo.

PALABRAS CLAVE: Andrés Piquer, Siglo, XVIII, Quina, Fiebres.

\section{SUMMARY}

In this article we analyze the historical figure of Andrés Piquer, offering a personal analysis about his medical work. We will take as a starting point the 1751 edition of his Tratado de las Calenturas (Treatise on Fever). We will use his allusions to fever and quinine as our two reflexive axes. In this analysis we will also refer to some of his Spanish and European - specially Frenchcontemporaries. We find Piquer clearly compromised with observation and experience as the only basis for medical knowledge. We think that this article is just a necessary first step in order to study, in future publications, the following editions of the Tratado, and so making possible a complete comparative analysis.

KEY WORDS: Andrés Piquer, $18^{\text {th }}$ Century, Quinine, Fever.

\footnotetext{
* Membre Correspondant, Centre Alexandre Koyré, EHESS, CNRS, MNHN, París.
} 


\section{EL SIGLO DE LAS TERCIANAS}

Al intentar ubicar históricamente el desarrollo de las distintas epidemias nos encontramos con la posibilidad de crear modelos temporales que nos permiten hacer una clasificación — si no totalmente nueva, sí al menos con una cierta diferencia - de los períodos en los que tradicionalmente hemos visto dividido el tiempo anterior a nuestra época. A veces, este intento puede coincidir, sin embargo, con la división cronológica habitualmente aceptada. Así, sin necesidad de situarnos en un sistema de imaginarios colectivos, encontramos cómo las representaciones de un azote epidémico como la malaria nos sirven de elemento clasificatorio temporizador. La imagen más nítida del fenómeno palúdico - la aparición de la fiebre - caracteriza el siglo XVIII de tal manera que se han convertido casi en sinónimos ${ }^{1}$.

La malaria ha sido presentada como la epidemia que en el siglo XVIII «reemplaza» a la peste - el gran azote del XVII, que tiene como última referencia la ciudad de Marsella en 1720 - antes de perder su papel de protagonismo social frente a la fiebre amarilla — en torno al paso del siglo XVIII al XIX —2

Al hacer referencia a este carácter de epidemia transitoria, o referente intermedio de esa trilogía a la que acabamos de aludir, no pretendemos olvidar, sin embargo, su presencia a lo largo de la historia; las epidemias palúdicas habían sido conocidas desde la antigüedad en el ámbito mediterráneo y a lo largo de la Edad Media habían afectado zonas pantanosas del litoral. Pero en el siglo XVIII tuvieron una especial importancia pues dieron lugar a «cifras alarmantes de morbilidad y mortalidad».

En España «las fiebres» o «calenturas» son un referente obligado durante todo el siglo XVIII. Los continuos accesos palúdicos, especialmente en la costa mediterránea, van a dar lugar a una atención especial de la parte de los médicos.

En cuanto al origen de esta mayor incidencia, Riera menciona cuestiones «geoclimáticas» — este autor apunta «la elevada pluviosidad»-, mientras que Peset y Pérez Moreda aluden a la relación de las fiebres con los cambios en los cultivos» ${ }^{3}$.

1 Ruffie, J.; Sournia, J. C. (1993), Les épidémies dans l'Histoire de l'homme, París, Flammarion, capítulo XI, pp. 225-250.

2 RierA, J. (1992), Capitulos de la medicina ilustrada española (Libros, cirujanos, epidemias y comercio de la quina), Valladolid, Universidad de Valladolid, Secretariado de Publicaciones, pp. 81-112, cita en p. 81. Estas líneas introductorias siguen, en sus aspectos históricos, la clarificadora presentación del tema que hace este autor.

3 Peset, J. L. (1987), Ciencia y libertad. El papel del científico ante la independencia americana, Madrid, CSIC, 1987, pp. 114-115. PÉREZ MoREDA, V. (1982), «El paludismo en 
Este aumento de la presencia del paludismo se puso de manifiesto en la región valenciana, zona que ha sido comúnmente considerada como endémica cuando se habla de la malaria ${ }^{4}$.

Los trabajos de Andrés Piquer son una referencia clave a la hora de abordar alguna de las cuestiones más relevantes de la medicina y de la salud españolas del siglo XVIII. Piquer, médico y catedrático de Anatomía en la Universidad de Valencia, ciudad donde estudió y publicó sus primeras obras, contaba con la experiencia procedente «de la frecuencia de la malaria en las zonas pantanosas de los arrozales valencianos» $\rangle^{5}$.

Las «calenturas» —utilizando la terminología del propio Piquer-van a conformar un campo de disertación al que el médico valenciano va a dedicar una parte importante de su reflexión sobre el fenómeno epidémico. En su Tratado de las Calenturas» ${ }^{6}$ va a precisar su pensamiento — enlazado con la observación/práctica/experiencia - en el tema de las fiebres y va a dedicar, asimismo, su atención al remedio más universal contra estas epidemias: la quina.

Fiebre y quinas van a ser dos términos no necesariamente complementarios en el discurso de Piquer, pero la relación entre ambos dará contenido a su doble propuesta teórica y práctica en el tema de las calenturas. La aparición en 1751 de su Tratado de las calenturas se puede considerar parte de una línea que se seguiría, entre otros, y de forma mucha más decidida, con José Alsinet y Cortada en 1763 y José Masdevall y Terrades en $1786^{7}$.

España a fines del siglo XVIII: la epidemia de 1786», en Asclepio, vol. XXXIV, pp. 305 ss., y PÉREZ MoREDA, V. (1984), «Crisis demográfica y crisis agrarias: paludismo y agricultura en España a fines del siglo XVIII», Congreso de Historia Rural. Siglos XV al XIX, Casa de Velázquez, Universidad Complutense, Madrid, p. 352, — para éste último sigo las citas de García Ruipérez, M. y SÁNCHEZ GonZÁLEZ, R. (1991), «La epidemia de tercianas de 1786 en la antigua provincia de Toledo», Asclepio, Vol. XLIII-1-. Véase también PeSET, J. L. y Peset, M. (1978), «Epidemias y sociedad en la España del Antiguo Régimen», Estudios de Historia Social, 4, pp. 7-28; y PeSET, M.; PESET, J. L. (1972a), Muerte en España, Madrid.

4 Lo que tampoco debe hacer olvidar que esta epidemia estuvo presente en muchas otras zonas del país. Ver, por ejemplo, el caso de la antigua provincia de Toledo en el trabajo de GARCÍA RuiPÉREZ Y SÁNCHEZ GONZÁLEZ (1991).

5 Riera (1992), p. 83. Peset, M. y PeSeT, J. L. (1972b), «Cultivos de arroz y paludismo en la Valencia del siglo XVIII», en Hispania, 121, pp. 277-375.

6 PiQuer, A. (1751), Tradado de las calenturas según la observación y el mecanismo, Valencia.

7 Alsinet, J. (1763), Nuevas indagaciones sobre la utilidad de la quina, Madrid. MASDEVAll Y TERRADES, J. (1786), Relación de las Epidemias de calenturas pútridas y malignas que en estos últimos años se han padecido en el Principado de Cataluña, y principalmente la que descubrió el año pasado de 1783, en Lérida, Llano de Urgel... Estas y otras muchas referen- 
El Tratado de las Calenturas es la última obra de las escritas por Andrés Piquer en su etapa valenciana, con el subtítulo de «según la observación y el mecanismo» — que desaparecerá en las ediciones posteriores - ${ }^{8}$. A finales de 1751 se traslada a Madrid, como médico de Cámara de Fernando $\mathrm{VI}^{9}$. En su primera obra publicada en Madrid, Piquer apuesta por las ventajas de la medicina experimental ${ }^{10}$, con una nueva actitud, decididamente antisistemática ${ }^{11}$. Con todo, en su primera presentación del Tratado de las Calenturas, vamos a encontrar a un Piquer basado en la observación y la experiencia - que serán los reclamos de sus alusiones a Hipócrates - que permanecerán posteriormente $^{12}$. Como ha señalado Alejandro Sanvisens, el mecanismo de la primera edición de la obra de Piquer sobre las calenturas, estaba «más en el nombre que en el contenido, en su totalidad» ${ }^{13}$.

La publicación de su Tratado en 1751 contó con la complicidad de Gregori Mayans, con quien había tenido una estrecha relación en sus años de Valen$\mathrm{cia}^{14}$. En esta primera edición es en la que hemos centrado nuestra atención.

cias, recogidas en RIERA, J. (1992); RUIPÉREZ; SÁNCHEZ (1984); y FONFRÍA, J., JiMÉNEZ, C.; FERNÁNDEZ J. (2001), «La corteza de la quina. Un remedio universal», en Estudios de Historia das Ciencias e das Técnicas, Pontevedra, Deputación Provincial, Servicio de Publicacións, tomo II, pp. 837-846.

8 PeSet i LlorCa, V. (1975), Gregori Mayans y la cultura de la Il-lustració, Valencia, Tres i Quatre, pp. 322 y 332. LOPEZ PIÑERO, J. Ma (1987), «Andrés Piquer y el hipocratismo en la España de la Ilustración», Introducción a Las epidemias de Hippócrates. Con observaciones prácticas de los antiguos y modernos, de Andrés Piquer, Madrid, Ministerio de Sanidad y Consumo, p. 26.

9 En Madrid Andrés Piquer tendrá un rápido ascenso, entrando a formar parte en 1752 del Tribunal del Protomedicato. En este año también fue designado vicepresidente de la Real Academia Médico-Matritense, de la que formaba parte desde 1739. PESET I LlORCA (1975), pp. 324 ss.; LÓPEZ PIÑERo (1987), p. 25; Archivo de la Real Academina Nacional de Medicina, Madrid, Legajo 2, documentos 25, 26 y 27.

10 PiQuer, A. (1752), De medicinae experimentalis praestantia, Madrid. Véase Ayala, J. M. (1996) Piquer (1711-1772), Madrid, Ediciones del Orto, pp. 28 y 29.

11 LÓPEZ PiÑERo (1987), p. 26.

12 Observación y experiencia «frente a todo sistematismo médico entroncado con concepciones filosóficas cerradas». MindAN, M. (1958), «Andrés Piquer y su contribución a la Historia de la Medicina», en Actas del XV Congreso Internacional de Historia de la Medicina, vol. I, Madrid, p. 172.

13 SanVisens Marfull, A. (1953), Un médico filósofo español del siglo XVIII: el doctor Andrés Piquer, Barcelona, CSIC, p. 94, nota 140.

14 Peset i LlorCA (1975), p. 322; la relación Mayans-Piquer, en pp. 230-392. 
El trabajo publicado por Andrés Piquer supone, por tanto, un punto de inflexión de obligada referencia en el intento de comprender el fenómeno de las fiebres en la España del siglo XVIII. Las sucesivas ediciones ${ }^{15}$ de su obra son un evidente reflejo de la positiva acogida de su trabajo, con un seguimiento notorio también fuera de las fronteras españolas, especialmente al otro lado de los Pirineos ${ }^{16}$.

A pesar de que muchas veces los supuestos se pueden identificar con los intereses ${ }^{17}$, y en el caso de Piquer también estarán presentes, no hemos abordado en la práctica esos intereses más que de una manera somera. Seguramente sea un trabajo necesario - quizás inminente- junto con nuestra reflexión sobre las propuestas de Piquer.

\section{DESCRIPCION E INDEFINICION}

La obra de Piquer aparece trazada en torno a la defensa de la «observación». En este sentido, las dudas y reparos atribuidas a la medicina son conse-

$152^{\mathrm{a}}$ edición en $1760 ; 3^{\mathrm{a}}$, en 1768 ; ambas corregidas. Sanvisens nos indica que tras su muerte se hicieron, aún en el siglo XVIII, otras dos ediciones a partir de la $3^{\mathrm{a}}$, en 1777 y 1778. SANVISENS MARFUll (1953), p. 62. Hemos localizado una $5^{\text {a }}$ edición, de 1788 -Facultad de Medicina de la Universidad Nacional Autónoma de México-.

16 Hubo traducción al francés - a cargo de Lamure-, así como una edición en Amsterdam. De las dos da cuenta Alejandro Sanvisens que precisa que la de Amsterdam se encuentra también traducida al francés, editada en 1776. SANVISENS MARFull (1953), p. 62. Acerca de su presencia» en Francia, Juan Bautista Peset sitúa a los médicos de la Escuela de Montpellier detrás de la traducción francesa del Tratado y mantiene que Philippe Pinel «copió...grandes trozos»; Peset, J. B. (1876), Bosquejo histórico de la medicina en Valencia, Valencia, Imprenta de Ferrer de Orga, p. 154. De su difusión en Francia hemos encontrado referencias en el Journal de Médecine... de Corvisart, una de las publicaciones períodicas médicas más influyentes de la época, lo que nos permite apreciar cómo aún a principios del siglo XIX el trabajo de Piquer era valorado en los círculos médicos franceses: «Traité des fièvres, de $\mathrm{M}$. André Piquer, Médecin de S.M.C.; Traduit de l'Espagnol en Français, par M***, D.M.M., sur la troisième et dernière édition de 1768; revu et corrigé par MM. C. et R., professeurs en médecine à Montpellier. Seconde édition. A Paris, de l'Imprimerie Economique, rue de la Harpe, No 117, An 9, 1801», en Journal de Médecine, Chirurgie, Pharmacie, etc, Prairial, An IX, pp. 281-283. También Chomel estaba atento a los trabajos de Piquer, como lo manifiesta en sus Éléments de pathologie générale, Paris, 1817 (La edición consultada, editada en Coimbre en 1839, según la edición francesa de 1824).

17 En el sentido que presentaba Bujosa en un interesante planteamiento epistemológico en el prólogo a su trabajo sobre Hernández Morejón, Chinchilla, Guardia y Comenge y Ferrer. BujosA, F. (1989), Filosofia e Historiografía médica en España, Madrid, CSIC, pp. IX-XV. 
cuencia de que o bien «se aplican poco los médicos a las observaciones» o bien «no las hacen con el cuidado que ellas piden». Sobre ellas deberá establecerse el «otro fundamento de la verdadera medicina»: el raciocinio ${ }^{18}$.

«Conocimiento» y comprensión son dos elementos que van a aparecer claramente diferenciados en el discurso de Piquer. Podemos conocer una enfermedad - e incluso esto será a veces algo fácil—, pero no es tan evidente el que podamos llegar a delimitar las características esenciales que van a permitirnos individualizar dicha enfermedad. Así, las calenturas son presentadas como algo fácil de conocer, aunque se precisa la dificultad de definirlas. El propio Piquer se muestra consciente de la limitación de esta presentación. Es por ello, quizás, que acude a los clásicos en un evidente intento de contestar de antemano a una previsible crítica a su trabajo ${ }^{19}$.

El recurso a la autoridad de Galeno e Hipócrates aparece entonces como algo natural que encontrará continuidad en su obra. En este sentido se recuerda la figura de Galeno, autor que, a pesar de que trató la cuestión de forma extensa, no abordó el tema de la definición. Alusión similar también la que se hace a Hipócrates. Aunque en este caso Piquer no cuestiona el punto de partida del médico griego de estar haciendo alusión más a síntomas que a verdaderas enfermedades. Tampoco es un obstáculo el hecho de que Hipócrates calificara con este término una serie de enfermedades sobre las que obligatoriamente tenía ideas distintas que los médicos de la segunda mitad del siglo XVIII y principios del XIX ${ }^{20}$.

La referencia a Galeno, apoyada en el juicio del médico español Vallés, contempla que no se definiera «porque tal vez conoció la dificultad que había en explicar la esencia de la calentura en una sola definición», pero también

18 PIQUER (1751), prólogo.

19 Situación que también se planteó en algún otro de sus trabajos. Ver, por ejemplo, las dudas y objecciones que le plantea el médico Jacinto Puig, a propósito de la Lógica Moderna — publicada por Piquer en 1747-. PUIG, J. (s.f.), Clave philosophica en la que se contiene una breve introducción a las disciplinas mathematicas y un fácil y breve compendio de la Lógica, o Philosophia racional, junto con una carta y dificultades propuestas al Doctor Don Andrés Piquer..., Madrid, — la carta aparece con fecha de 17 de abril de 1752 - pp. 133, 134 y I-XVIII.

20 Artículo «Fièvre», en ADELON et al. (1822), Dictionnaire abrégé de Sciences Médicales, Panckoucke, París, tome septième, p. 369. En sentido similar encontramos a Boisseau: «on ne trouve dans les écrits qui portent le nom d'Hippocrate, rien qui resemble à la manière dont on considère aujourd'hui ce qu'on appelle les fièvres... les Hippocratides...n'ont jamais entendu désigner une classe, un genre, une espèce de maladie en général, mais seulement un symptôme...»; BoISSEAU, F.-G. (1824), Pyrétologie physiologique ou Traité des fièvres considerées dans l'esprit de la nouvelle doctrine médicale, París, pp. 52 ss. 
admite que quizás Galeno «debió hacer juicio, que son vanas las definiciones de aquellas cosas que son manifiestas por sí mismas». Aunque Piquer parece decantarse por la evidencia en el tema de las fiebres, su posición aparece precisada cuando escribe: «iqué importa que se ignore en qué consiste la esencia de la calentura, como se sepa conocer cuándo la hay y de qué manera ha de curarse?». Por lo tanto, aunque el médico no conozca la esencia del mal, podrá conocerlo y también curarlo si conoce los caracteres que le son propios ${ }^{21}$.

Este planteamiento frente a la enfermedad obliga a encontrar un camino distinto que permita ocupar el espacio de definición que se nos presentaba inabordable. En el caso de Piquer, va a ser la «descripción» la que se encargue de ello, la que deberá reflejar los caracteres propios e inseparables que nos servirán de elementos de identificación y de diferenciación. En referencia a las calenturas estos caracteres van a ser: «pulso acelerado», "calor más intenso» $\mathrm{y}$ «acciones de la vida dañadas». Estas son las circunstancias determinantes para considerar que una persona tiene calenturas, «pues es imposible haber estas tres cosas en un sujeto sin que la calentura exista» ${ }^{22}$.

En cuanto a la división de las calenturas Piquer presenta tres grandes grupos: diarias - las que duran veinticuatro horas, más o menos-, pútridas - las que «suponen putrefacción en los humores»-y héticas — «aquellas que son lentas, largas, continuas, y necesariamente producen grande extenuación del cuerpo, y siempre nacen de otra enfermedad que las fomenta»-. A partir de estos grupos se presentan numerosas divisiones ${ }^{23}$. No nos detendremos ahora en los distintas tipos de fiebres que considera Piquer. Más interesante nos parece abordar la manera en la que nos presenta las causas generales de las calenturas, pues a través de ello nos va a introducir también en el método general de su construcción conceptual de la enfermedad ${ }^{24}$.

\section{EL ORDEN NATURAL}

Piquer identifica salud con orden, y enfermedad con desorden. Así, considera que el estado natural del hombre es estar sano. En él, las distintas dispo-

21 Postura práctica que encontraremos también en el mundo de las fiebres al intentar esclarecer la acción de la quina.

22 PIQUER (1751), pp. 1-4.

23 Ibidem, pp. 5 y 6.

24 Sobre las influencias médicas de Piquer véase SANVISENS MARTull (1953), pp. 150154. Sobre su pensamiento filosófico AYALA (1996), pp. 25-32; SANVISENS MARTULL (1953), pp. 161-181. 
siciones que se necesitan para la vida están ordenadas, al tiempo que se cumplen «debidamente» las leyes de los movimientos. La enfermedad, en cambio, va a ser un indicio de que las disposiciones o movimientos no están bien ordenados. En este razonamiento, «será tanto más peligrosa la dolencia cuanto mayor fuese el desorden ${ }^{25}$.

Habiendo señalado que las calenturas tenían tres caracteres propios - pulso acelerado, calor más intenso y daño en las acciones vitales - , todo aquello que pudiera causar estos efectos en el cuerpo humano estaría también en disposición de producir calenturas. Las causas pueden ser numerosas, pero Piquer las reduce a dos clases: la naturaleza misma del hombre y las demás cosas que puedan alterar esta naturaleza dando lugar a los efectos citados. Por lo tanto, la naturaleza aparece como la «causa universal, próxima, inmediata y necesaria de todas las calenturas». Y ¿qué entiende Piquer por «naturaleza»? Para él se trata del «principio y causa material y física de las operaciones humanas». Pero no se trata de un principio unitario, sino «en el concurso y agregado, mutua harmonía, y correspondencia de todas aquellas cosas que son necesarias para la constitución del cuerpo humano». Por ello, en cuanto objeto de la medicina, la naturaleza del hombre es el conjunto de sólidos y líquidos que componen el cuerpo humano con el concurso del orden y la correspondencia que deberá haber entre ellos.

La cuestión que se plantea a continuación es la de saber cuándo la naturaleza actúa bien en las enfermedades - el médico seguirá estos movimientos - y cuándo no —el médico los reprimirá-. Todos los movimientos de la naturaleza humana, en cuanto a su conservación, se hacen según las leyes del Creador. El médico observará estos movimientos, tanto en la salud como en la enfermedad, lo que le servirá de axioma para fundamentar su discurso.

Este modelo de «Mecanismo» no basta, sin embargo, para explicar las causas finales de las calenturas. Es por ello que Piquer considera que, además de las leyes generales del movimiento, en el cuerpo humano también funcionan unas leyes particulares - las que algunos denominan «Mecanismo propio del hombre» y otros «principio vital»- La consideración que hace de este «principio» nos presenta a un Piquer que se encuentra más cerca de la practicidad propia del carácter de las nuevas ideas del siglo XVIII que del pensamiento sistemático del que aparentemente parecería formar parte: «para el uso que en la Medicina se puede hacer de estas cosas, basta la atenta observación de los efectos que de él proceden; porque importa poco que se ignore el mecanismo especial del hombre, con tal que se sepan los efectos que de él dima-

25 PiQUer (1751), pp. 10-22. 
nan, los tiempos en que obra y la correspondencia y demás cosas reparables que hay en ellos y les pertenecen».

Aquí se entiende la mentalidad antisistemática de Piquer, que «no significa falta de método y sistema en su investigación y exposición médico-filosófica» sino su apuesta por unos principios basados en la comprobación empírica ${ }^{26}$.

Piquer confiesa «su ignorancia sobre la causa íntima de las fiebres, desconoce como Sydenham el motivo de la periodicidad palúdica y expone a menudo sus dudas con leal franqueza» ${ }^{27}$. Ello no impedirá que intente buscar una explicación e intente identificar, al menos, la causa aparente de las calenturas.

En el modelo presentado por Piquer, con continuas alusiones hipocráti$\operatorname{cas}^{28}$, es la naturaleza la que «halla los caminos que necesita para saber lo que es saludable al cuerpo», es decir, que es la naturaleza «la que cura las enfermedades» y ella misma «busca los caminos que son necesarios para vencerlas». Por tanto, consecuentemente con lo anterior, será la naturaleza la causa más próxima de las fiebres. Y esta naturaleza o disposición mecánica del cuerpo humano será simpre la misma, con la única diferencia que en la salud el mecanismo está de un modo y en la enfermedad, de otro distinto.

Dando por supuesto que la naturaleza es la causa de las fiebres y que su mecanismo está alterado cuando se producen, la siguiente cuestión deberá dirigirse evidentemente hacia las causas que alteran la disposición mecánica del cuerpo humano y que conllevan la aparición de la calentura. De nuevo con Hipócrates, Piquer señala el «aire» como la «más universal y más eficaz causa de las calenturas $\gg{ }^{29}$. Las alusiones a la fiebre y las pestes parecen evidentes - relación que había estado presente a todo lo largo del siglo XVIII y que aún tendrá vigencia en los inicios del XIX - ${ }^{30}$. La referencia al aire como vehículo transmisor va a estar unida a la cercanía de balsas o lagos que - especialmente en el caso de las fiebres tercianas - va a ser determinante ${ }^{31}$.

26 SANViSEnS MARFUll (1953), p. 150.

27 Peset y Cervera, V. (1934), Andrés Piquer: (recuerdo apologético de la excelsa figura del siglo XVIII), Valencia, Imp. Hijo de F. Vives, p. 39.

28 Sobre el hipocratismo de Piquer: SANVISENS MARfull (1953), pp. 80-84; LÓPEZ PIÑERo (1987), pp. 28-33; ÁNGEL Y ESPINOS, J. (1998) «Andrés Piquer et la tradition hippocratique dans l'Espagne du XVIIIe siècle», en Vesalius. Revue officielle de la Société Internationale d'Histoire de la Médecine, vol. IV, $\mathrm{n}^{\mathrm{o}}$ 1, Juin, pp. 31-34. Laín incluye a Piquer en el grupo de hipocráticos de «actitud ahistórica»; LAÍn ENTRALGO, P. (1998), La historia clínica. Historia y teoría del relato patográfico, Madrid, Editorial Triacastela, p. 49 ( $1^{\circ}$ ed. en 1950).

29 PIQUER (1751), p. 15.

30 Chomel, A.-F. (1821), Des fièvres et des maladies pestilentielles, París.

31 PiQuer (1751), p. 238. Sobre «el aire y la amenaza pútrida» véanse las interesantes páginas de Corbin, A. (1997), Le miasme et la jonquille, París, Flammarion, pp. 11 ss. (1 $1^{\mathrm{a}}$ éd. 
Las alusiones al aire aparecerán en los distintas fiebres. Así, «las calenturas ardientes casi todas nacen del aire» ${ }^{32}$; el aire es «casi siempre» la causa de las sinocales $^{33}$; la causa de las malignas «es un veneno de especial naturaleza que va con el aire» ${ }^{34}$; «ninguna causa es más eficaz para producir estas calenturas, que el aire, en especial las tercianas malignas, que se hacen tales por las malas influencias que el aire comunica a los cuerpos que están dispuestos a padecerlas $\rangle^{35}$.

El argumento causal del aire le permite a Piquer, además de explicar la llegada de la fiebre, avanzar una fundamentación sobre la aparición de distintas fiebres rompiendo unos hipotéticos ciclos anuales: «es verdad que no todos los años son las calenturas de una misma índole; pero esto nace de que tampoco es de una misma calidad el vicio del aire». Esta constatación irá unida a la modificación de efectos que el aire produce «según las disposiciones que encuentra en los cuerpos» ${ }^{36}$.

Una vez determinada la vía de llegada del mal Piquer se cuestiona de qué modo el aire produce la calentura. Aquí la experiencia deja de ser útil, al no poder ser percibido por los sentidos. Habrá que ir, por lo tanto, a los efectos producidos. Es decir, frente al «a priori» de la experiencia aparece el «a posteriori» de lo causado ${ }^{37}$. La parte considerada venenosa del aire va a actuar causando «contracciones espasmódicas en los sólidos» — como el corazóny «alteraciones en los líquidos», produciéndose así la calentura.

Aceptando el razonamiento de esta explicación y su consiguiente conclusión final, parece consecuente que junto al aire aparezcan «alimentos», «me-

en 1982). Sobre la influencia siempre presente de los pantanos, modificando la tendencia a asignar al aire un protagonismo que muchas veces no ha tenido: DUMAS (1791), «Discours préliminaire», Cours complet des fièvres par feu de M. Grimaud, Paris, p. XXVII; también: BAILly, E. M. (1825), Traité anatomico-pathologique des fièvres intermittentes, simples et pernicieuses, París, pp. 122 y ss. La identificación de los lagos con las fiebres palúdicas ha dado lugar a un importante debate dentro de la historia de las mentalidades; JEANFILS, E. (1986), «L'evolution de la perception des marais du point de vue culturel et institutionnel», en L'impact des activités humaines sur les eaux continentales, Société Hydrotechnique de France, París, Question I, rapport no 1 et fascicule général, pp. 30-31.

32 PIQUER (1751), p. 32.

33 Ibidem, p. 105.

34 Ibidem, p. 151.

35 Ibidem, p. 238.

36 Ibidem, p. 16. En p. 21 añade: «la disposición de los cuerpos hace mucho para que el aire obre en ellos con mayor o menor actividad».

37 Ibidem, p. 22. 
dicinas», o «cualquier otra cosa» que puedan producir también calenturas, en la medida que pueden actuar igualmente sobre los líquidos o partes sólidas del cuerpo ${ }^{38}$.

Causa, modo y efecto aparecen, por tanto, como los tres ejes sobre los que Piquer articula su explicación de cómo se producen las fiebres. A partir de aquí, y usando las descripciones como «único medio que hay para representar las enfermedades», Piquer abordará los distintos tipos de calenturas que él privilegia: ardientes, sinocales o continentes, malignas, semitercianas, cotidianas o mesentéricas, diarias, tercianas y cuartanas.

\section{LA ACCIÓN DEL MEDICAMENTO}

Ademas de la singularidad de Andrés Piquer en el tema de las fiebres, conviene que nos centremos tambien en las referencias que este autor hace de la quina.

A pesar de que la quina era conocida desde más de un siglo antes ${ }^{39}$, y que había tenido una favorable acogida en Europa ${ }^{40}$, en el siglo XVIII seguían proponiéndose remedios clásicos. Así, encontramos cómo algunos autores hacían aparecer en una posición de privilegio la cebada y las claras de huevo - siguiendo la receta hipocrática-, y otros en los que también intervenían el salvado de trigo, la harina de avena, la calabaza, la «goma amoníaco», el vino, el antimonio o, especialmente para los niños, las almendras. Bien es verdad que junto a ellos también ocupaba un lugar la quina, pero lejos del papel destacado que sí se le estaba dando desde otros círculos ${ }^{41}$.

En el caso de Piquer, especial dedicación a la quina nos encontramos en el capítulo referido a la curación de las calenturas ardientes ${ }^{42}$. Y tanto más significativa es la alusión a la planta americana en cuanto que Piquer va a desarro-

\footnotetext{
38 Ibidem, p. 23.

39 Hernando, T. (1982), «La quina en el siglo XVII», en Dos estudios históricos (Vieja y Nueva Medicina), Madrid, Espasa-Calpe, pp. 171-223.

40 Como queda de manifiesto especialmente en algunas de las obras que aludían a las enfermedades comunes: Guide ou Manuel dans le traitement des maladies les plus graves et les plus fréquentes, París, 1777, pp. 69 y ss.; TISsOT, S. A. (1782), Avis au peuple sur sa santé ou Traité des maladies les plus fréquentes, París, tomo I, pp. 274-287. En algunas se recomienda incluso para todo tipo de fiebres: La médecine et la chirurgie des pauvres, París, 1758, pp. 283-289.

41 Curbo Semmedo, J., (s.f. pero c. 1731), Secretos médicos y chirurgicos del doctor...., traducidos de lengua vulgar portuguesa en castellana por el Doct. D. Thomas Cortijo Herráiz..., Madrid, pp. 12 y ss., y 76.

42 PIQUER (1751), pp. 66-69.
} 
llar aquí todo su escepticismo frente a los remedios: confianza en la acción de la naturaleza - de marcada herencia hipocrática - y ataques a los sabios aplicadores de medicina. Así, tras señalar una división entre enfermedades agudas - «que andan acompañadas de muy graves síntomas, los cuales por lo común son breves, y suelen terminarse dentro de cuarenta días»— y crónicas - «las que se alargan mucho»- concluye que «es indubitable, que la naturaleza es la que curas unas y otras, y las medicinas en tanto aprovechan, en cuanto socorren y ayudan para que pueda expeler las causas de las dolencias»». La naturaleza va a aparecer no ya como algo necesario o importante sino como decididamente imprescindible, pues donde faltara ella «no hacen los medicamentos ningún efecto» ${ }^{43}$.

Piquer recuerda los dos grupos diferenciados que encontramos sobre este tema. De un lado señala a "Gedeon Harveo» - Gideon H. Harvey-, que «quiso que los médicos hiciesen muy poco, o nada, sino solo observar a la naturaleza y dejarla sin medicinas, suponiendo que ella sola ha de hacer la curación». En este grupo incluye al Dr. Boix —el autor de Hipócrates defendido - también partidario de esta línea pero menos radical que Harvey. En el lado contrario sitúa a los que «quieren hacerlo todo con medicinas, como si la curación la hubiesen de ejecutar ellos solos, sin dejar nada a la naturaleza». En este grupo, claramente denostado por Piquer, son aludidos «algunos autores de Farmacopea» y un grupo muy determinado al que hacíamos alusión unos párrafos más arriba: los químicos. Para Piquer, estos últimos, «con sus Panaceas, y Arcanos», son «extremadísimos» en seguir esta tendencia de utilización profusa de medicinas.

Nos encontramos, por tanto, con una clara alusión a la diferenciación de actitudes de dos grupos - médicos y químicos- que cobrará cuerpo a finales de siglo y especialmente a principios del siguiente.

Frente a estas dos posturas extremas Piquer pretende tomar un camino intermedio, señalando también en esta ocasión que es la naturaleza la que cura las enfermedades y que el trabajo de los médicos consiste en adivinar el curso de esta acción y ayudar a llevarla a cabo. Esta división aparente del trabajo queda derrumbada cuando tiene que tomar opción: "Y si hubiera yo de decir cual de los dos extremos que acabamos de proponer es el peor, siempre ten-

43 «Finalmente, para llevar acertadamente la curación, así de esta calentura como otras agudas, es menester observar constantemente la máxima de que la naturaleza es la que las cura; y que el médico no ha de apresurarse con muchas medicinas, porque pocas y escogidas y aplicadas según el destino que la naturaleza requiere, harán más provecho, que la multitud que hallamos hacinada en muchos libros y farmacopeas». Ibidem, p. 99. 
dría por mucho más perjudicial al linage humano la opinión de los que todo quieren curarlo con muchas y repetidas medicinas, que la de aquellos que no quieren que se use ninguna».

La división entre enfermedades agudas y crónicas cobra ahora mayor sentido. En las primeras se necesitan pocas medicinas para curarlas. El hecho de ser breves se interpreta de dos maneras. Por un lado, la falta de necesidad: como esta enfermedad se acaba rápido, no es preciso que la naturaleza haga uso de medicamentos. Por otro, una interpretación de causa/efecto: la naturaleza obra de manera muy eficaz «y con la actividad de sus movimientos trabaja mucho en expeler y arrojar del cuerpo las causas de la dolencia». Con lo que la brevedad de la enfermedad sería debido precisamente a la brillante actuación de la naturaleza. Siguiendo este último razonamiento nos encontramos una mayor necesidad de medicamentos en las enfermedades crónicas, pues en ésta la naturaleza obra más lentamente.

Este es el marco que sirve para que la quina haga su aparición en el caso de las «calenturas ardientes», con el aval de la experiencia y uso que de ella testimonia Piquer.

\section{EL REMEDIO DE LA QUINA}

Observación y raciocinio son los dos referentes que Piquer acepta para la introdución del uso acertado de los remedios. La importancia del raciocinio queda patente en el hecho de que gracias a él «también» se deduce «la aplicación que puede hacerse de las medicinas en las enfermedades». Pero son una importancia y una consideración secundarias pues considera que en él aparecen «más engaños y equivocaciones que en la observación». Para ser aprovechado en medicina, el raciocinio «debe siempre fundarse en las operaciones de la naturaleza, de modo que el razonamiento del médico ha de ser enteramente conforme con lo que la naturaleza ejecuta».

Queda evidenciada, por lo tanto, la valorización de la observación, en la que ha de apoyarse la elección de los posibles remedios: «Llevaremos pues por máxima fundamental para nuestra curaciones, preferir siempre a cualesquiera otros, los remedios cuya eficacia consta por observaciones ciertas, y por raciocinios naturales deducidos de lo que la misma naturaleza enseña». Hagamos notar, además, la precisión que Piquer hace sobre la observación: que sea «cierta», que no vale cualquier tipo de observación, sino aquella realizada con fundamento: «y de este modo de aplicar las medicinas nunca engañaría si las observaciones estuviesen bien hechas, porque de las cosas que 
constan por observación fiel y segura, se tiene evidencia». Y en esta declaración de principios sobre las observaciones positivas encuentra un claro acomodo su apuesta por la quina: «por haber observado los hombres que el opio quita los dolores, y la quina las calenturas, se aprovechan de estos remedios para quitar estas dolencias». Riera ha señalado - haciendo referencia al siglo XVII - que «el empleo de la quina como recurso médico fue el resultado de la observación clínica, siguiendo la lógica de los misioneros jesuítas $\rangle^{44}$. En el caso del discurso de Piquer aparece claramente que es la observación, y en este caso una observación fiable y segura - la quina elimina la fiebre- el fundamento de su defensa de las propiedades de la planta americana.

Las fiebres aparecen como algo fácil de conocer pero difícil de definir. Es decir, la descripción de las calenturas se presenta como algo abordable, frente a la dificultad que surge al pretender establecer precisiones delimitatorias. Definir la enfermedad - como síntoma o como patología - se convierte, por lo tanto, en una tarea casi inabordable. En el listado inmenso de distintas fiebres que aparecen en cualquier nosología —al igual que en el Tratado de Piquer - encontramos seguramente una primera respuesta a esta imposibilidad de definir el mal al que se está haciendo referencia. La observación cobra por tanto protagonismo no sólo como elemento que suministra los datos previos a la elaboración de una teoría sobre la enfermedad, sino como el verdadero laboratorio de creación de un discurso contra dicha enfermedad. La historia clínica $-\mathrm{y}$ las referencias hipocráticas para el caso de Piquer vuelven a evidenciarse - aparece de esta manera como un recurso necesario - más que complementario-, como la base empírica de un futuro modelo teórico.

Es en este sentido donde cobra importancia la observación, tanto sobre las propias calenturas, como cuando se trata de aplicar el remedio de la quina. Un medicamento que aparece, además, con un uso limitado. Es decir, se trata de un medicamento/remedio contra la fiebre, en cuanto que sirve para curar el mal, pero no aparece signo alguno que pudiera hacernos pensar en medidas preventivas o de precaución ${ }^{45}$.

Hicimos mención unas líneas más arriba a la aparición de la quina en el caso de las calenturas ardientes. En las demás fiebres señaladas por Piquer la alusión al remedio americano será desigual. Si en el de las malignas destaca por su ausencia — frente al protagonismo de la sangría—, la volveremos a

44 RIERA (1992), p. 82.

45 En el mismo sentido que ha sido señalado para el caso francés por Georges Vigarelo. Vigarelo, G. (1993), Le sain et le malsain. Santé et mieux-être depuis le Moyen Age, París, Seuil, p. 134. 
encontrar - si bien en una posición claramente secundaria - en el caso de las semitercianas $^{46}$, y aludiendo a la circunstancia de que dicha calentura se hiciera intermitente.

Va a ser, efectivamente, en el caso de las tercianas cuando la utilización de la quina adquiera un plano destacado - si bien siempre detrás de una primera curación con sangrías y vomitivos—47. La quina aparece, entonces, no solo como el remedio más eficaz, sino como «el único» frente a esta enfermedad. Y en su utilización no se mezclará con otras medicinas - salvo casos puntuales - porque - tomando como apoyo una vez más la experiencia«los polvos de la quina bien escogida, de por sí solos hacen mejores efectos».

En el caso de las tercianas malignas la utilización de la quina es colocada por Piquer en primer lugar, antes incluso de hacer sangrías ni dar vomitivos. Con ello muestra una vez más su confianza en este remedio que cura a los enfermos, sin otro tipo de prevenciones. Así, frente a la posibilidad de que los médicos se entretengan en dar medicinas evacuativas - antes de que se mueran los enfermos - Piquer recomienda la quina, y estos enfermos se curarían «con tal que desde luego se les de quina sin prevención ninguna». Piquer hace uso en este caso de su propia experiencia: «De una vez doy yo media onza de quina en estos casos, y vuelvo a repetir la misma cantidad dentro de algunas horas, hasta que vea que la accesión de la terciana no viene, como regularmente suele suceder. Y después de haberse ya quitado, hago tomar al enfermo todos los días un papel de quina de dos dragmas hasta que cumpla una onza» ${ }^{48}$.

La quina aparece recomendada también en el caso de las cuartanas - dado que comparte «causa» con las tercianas-. En la referencia a las cuartanas también se constatan sus buenos efectos, pero teniendo en cuenta que con cualquier «leve motivo vuelven despúes de ella» ${ }^{49}$.

Cuando las fiebres intermitentes - tercianas o cuartanas - se vuelven continuas, la dosis de quina debe aumentar considerablemente, con el objetivo de impedir el peligro que este paso puede conllevar. Aún siendo ya continua, y dado el origen como intermitente, la utilización de la quina será siempre pertinente ${ }^{50}$.

\footnotetext{
46 PiQUeR (1751), p. 210.

47 Ibidem, pp. 240 y ss.

48 Ibidem, p. 242.

49 Ibidem, p. 245.

$50 \quad$ Ibidem, p. 246.
} 


\section{IDENTIFICACION}

La identificación de la quina va a ser la asignatura pendiente. El no tener los elementos precisos de determinación será una de las causas más decisivas a la hora de presentar posturas a favor o en contra de la planta ${ }^{51}$. La utilización de una gran variedad de términos es un reflejo asimismo de la propia confusión que se dio con este remedio ${ }^{52}$.

La agrupación conforme a la situación geográfica - dado que «respetan bastante los lazos naturales por la circunscripción de especies en centros relativamente limitados»— va a seguir siendo un elemento clasificador hasta bien entrado el siglo XIX. El criterio de la apariencia exterior - profusamente utilizado en las primeras presentaciones - va a convivir con el basado en las diferencias de composición química. Con todo, desde dos de los referentes básicos de la medicina española en el siglo XIX, como son el francés y el alemán, vamos a seguir encontrando la constatación de esta indefinición. Así, en 1874 aún podemos encontrar afirmaciones desde los círculos franceses, como la de Goubler: «una división más precisa y segura que ésta, fundada sobre los caracteres botánicos de las especies no es posible todavía por la incertidumbre de las determinaciones ${ }^{53}$. En un sentido similar también escribirá Husemann: «se ha extendido el conocimiento de los árboles de la quina hasta el punto de que sepamos existen al menos 3 y quizá muchas docenas de especies del género cinchona...», al tiempo que señalará la presente confusión entre las distintas cortezas ${ }^{54}$.

51 Problema que había estado presente en los intereses de los grandes botánicos de la época y del que los españoles tuvieron un protagonismo destacado. FERNÁNDEZ PÉREZ, J. (1998), «The relations betwenn Linnaeus and Mutis, The problem of the determination of the Cinchona trees», en Carl Linnaeus and enlightened Science in Spain, Madrid, Consejería de Educación y Cultura, CAM, pp. 69-102.

52 Como ya señalamos, por ejemplo, en el caso de los considerados «principales» diccionarios franceses de Historia Natural de la segunda mitad del siglo XVIII y primeras décadas del XIX; FríAs NúÑEZ, M. (1998), «Problemas terminológicos en la identificación de 'La quina americana' (1764-1828)», en Boletín de la Real Sociedad Bascongada de los Amigos del País, tomo LIV-1, pp. 53-61.

53 Goubler, A. (1877), Comentarios terapéuticos del Codex Medicamentarius, o sea Historia de la acción fisiológica y de los efectos terapéuticos de los medicamentos, (2 ${ }^{\text {a }}$ edición en español; la edición original francesa es de 1868), Madrid, p. 342.

54 Husemann, T. (1878), Manual de Materia Médica y Terapeutica, escrito para estudiantes y prácticos, con comentarios a la Farmacopea Germánica, Madrid, t. III, pp. 96 y 97. 
A pesar de la cuestión de la identificación, el posible problema de los componentes de la quina no se presenta como tal, porque lo que importa son los resultados. La ignorancia sobre la actuación del remedio $-\mathrm{y}$ en consecuencia el desconocimiento de la causa de su efectividad - se va a mantener durante su difusión en Europa. ${ }^{55}$ Ello no impedirá, como hemos señalado en otro trabajo, la aceptación de la quina tanto desde los medios oficiales e institucionales - dirigidos predominantemente a un público «médico»o entendido en cuestiones de salud - como desde las publicaciones dirigidas a sectores más populares ${ }^{56}$.

La cuestión de los componentes de la quina y su modo de actuación seguirá sin resolverse aún durante un largo tiempo — que no se clarificará hasta los trabajos de Caventou y Pelletier ${ }^{57}$ - Con todo, la ignorancia de la manera de actuar y especialmente de la causa de su efectividad va a continuar, y ello no va a ir en detrimento del prestigio de la planta. Esta constatación será relativizada desde la posición de que «poco importa su modo acción» sino lo que interesa en realidad es que cure y termine con las fiebres ${ }^{58}$.

La quina queda a salvo de cualquier acción negativa. En este sentido, y a propósito de las calenturas cotidianas o mesantéricas, Piquer reconoce que el uso de la quina puede producir una inflamación interna e incluso la muerte. Ahora bien, esto se debería a un mal uso de la quina y en ningún caso supone un cuestionamiento de sus propiedades. Los posibles trastornos han de entenderse - dice Piquer - «del mal uso de la quina o de la demasiada abundancia y tiempo poco a propósito en que algunos la propinan», concluyendo con una nueva apuesta por la observación, al tiempo que introduce una llamada al buen hacer del médico - «porque por repetidas observaciones sabemos que la quina acaba de quitar las calenturas mesentéricas cuando son muy porfiadas, y el médico ha hecho las diligencias previas que pide este remedio» ${ }^{59}$.

55 Nouveau Dictionnaire d'Histoire Naturelle apliquée aux arts, principalemente à l'Agriculture et à l'Economie rurale et domestique...,(An XI-1803), Déterville, París, p. 137.

56 FRíAs NúÑEZ, M. (2001), «Acerca de la utilización de la quina americana», Estudios de Historia das Ciencias e das Técnicas, Pontevedra, tomo II, pp. 847-853.

57 Pellentier ET CAVENTOU (1821), Analyse chimique des quinquinas, suivie d'observations médicales sur l'emploi de la quinine et de la cinchonine, Paris.

58 FRÍAS NúÑEZ, M. (2002), «La matière médicale américaine: le quinquina et les Dictionnaires d'Histoire Naturelle», en Biological an Medical Sciences (D. BUICAN and D. THIEFFry Eds.), Liège, Brepols Publishers, p. 90.

59 PIQUer (1751), p. 230. 


\section{A MODO DE CONCLUSIÓN}

Filosofía y medicina - con el referente del pensamiento médico- han llevado un camino de encuentros a lo largo de la historia, pero un camino evolutivo y que en gran medida es un reflejo de los cambios de mentalidades ${ }^{60}$.

Piquer intenta tomar distancia de los prejuicios filosóficos — que él mismo destacaba en su crítica a la medicina árabe - tomando partido por la «sensata experiencia». Resulta en principio llamativo, como ha señalado Manuel Mindán, «que mientras por un lado creía Piquer que la filosofía era necesaria al médico, hasta tal punto que él mismo escribió sus tratados de filosofía pensando sobre todo en ayudar a los estudiantes de medicina», mantenía por otro lado que «la filosofía puede ser gravemente perjudicial a esta ciencia...Pero - concluye Mindán - el comprometerse con un sistema filosófico cerrado es peligroso para el médico, porque llena de prejuicios y le hace preferir las construcciones fantásticas del ingenio a las realidades indefectibles de la experiencia y al buen sentido del juicio». ${ }^{61}$ Sanvisens venía a concluir que la «medicina hipocrática de Piquer no pretende fundamentos filosóficos inmediatos» y que, además, «aunque deba complementarse y fundamentarse bien, la Medicina no es Filosofía; aunque entienda la naturaleza, como Piquer, de un modo integral»». ${ }^{62}$ Quizás sea Jorge M. de Ayala, quien aporte otro prisma cuando señala, de una manera muy esclarecedora, más allá de la simple constatación - a partir del estudio de la Lógica piqueriana - que Piquer no era filósofo sino médico de profesión. Esto haría que «en lugar de concentrarse en el análisis de la potencia operativa de la razón analizando la naturaleza de los juicios» — como hará Kant—, se limitara «al análisis de la experiencia como origen o fuente de las verdades». Este análisis permite a Ayala recoger la cita del propio Piquer de que la experiencia «es la fuente de importantísimas verdades, entendidas éstas no en el sentido de 'operación realizada de modo controlado sobre dichos hechos, en espera de una respuesta previamente determinada', sino de observación de un hecho por los sentidos»». ${ }^{63}$

Hemos hecho hincapié en otro lugar en el proceso de individualización que a finales del siglo XVIII y especialmente a principios del siglo XIX represen-

60 Tubiana. M. (1995), Histoire de la pensée médicale, París, Champs-Flammarion.

61 MiNDAN (1958), p. 172.

62 SANVISENS MARFUlL (1953), pp. 96 y 97.

63 PiQuer, A. (1747), Lógica Moderna, o Arte de hallar la verdad, y perficionar la razón, Valencia. Ayala (1996), pp. 40 y 41. 
ta la utilización de la quina en el caso de las fiebres palúdicas ${ }^{64}$. La propuesta de Piquer puede considerarse una decidida aportación desde los círculos españoles a esta nueva conceptualización que se estaba forjando en los medios científicos europeos. Proyección que se recogería en los grandes diccionarios científicos de referencia de la época - tanto de Medicina como de Historia Natural- así como en publicaciones y trabajos particulares de algunos de los médicos con más prestigio del momento.

Philippe Meyer ha insistido recientemente en que el progreso del pensamiento médico, al igual que el conjunto del progreso científico discurre de manera discontinua ${ }^{65}$. Meyer utiliza el ejemplo de la pieza de cristal — que necesita una base sólida, sobre la que ir colocando las sucesivas capas-. Al abordar la reflexión de Piquer en su Tratado de las calenturas estamos hablando de un esfuerzo de integración de la medicina española, en un referencial de epistemología general, pero al mismo tiempo, de un necesario posicionamiento de una manera de entender la práctica médica. En este sentido, sin recurrir a denostadas visiones teleológicas, sí estamos en la senda de precisar interesantes y motivados antecedentes de muchas de las incorporaciones - entre ellas, el experimento ${ }^{66}$ - que se integrarán en los conocimientos sobre la salud y la enfermedad en la medicina contemporánea. Propuesta de Andrés Piquer que, junto a sus referencias a las autoridades, sus citas galénicas y su constante apoyo en Hipócrates, se muestra claramente apostando por la observación y la experiencia - y aquí la presencia de Sydenham ${ }^{67}$ es determinante - como base del conocimiento médico ${ }^{68}$.

64 FRÍAS NúÑEZ (2002).

65 MeYer, P. (1998), Leçons sur la vie, la mort et la maladie, París, Hachette, p. 85.

66 Jorge M. Ayala ha insistido en que Piquer no era un «experimentalista» en el sentido contemporáneo del término. De hecho «desconfía de los experimentos hechos en los laboratorios». Pero también ha señalado su apoyo en el experimento, como «hecho natural que observamos por los sentidos y se pinta en la imaginación». AYALA (1996), pp. 28 y 29.

67 Sydenham, T. (1676), Observationes Medicae. Una edición francesa de sus trabajos, en Sydenham, T. (1816), Oeuvres de médecine pratique de Thomas Sydenham, Montpellier.

68 Elementos y referencias presentes en la obra de Piquer, que seguirán teniendo protagonismo en los medios oficiales de la medicina un siglo después. Así, por ejemplo, junto a la insistencia en la observación, encontramos la aceptación de las propuestas de Sydenham sobre la quina y las fiebres intermitentes en las lecciones clínicas llevadas a cabo por Britonneau en el Hospital de Tours en la primera mitad del siglo XIX: «Essai clinique sur les fièvres intermittentes», en Journal des connaissances médico-chirurgicales, publié par Trousseau, Lebaudy et Gourand, $n^{\circ}$ V, janvier, 1834, pp. 135-138. 